\title{
Strain partitioning in front of an obliquely convergent indenter
}

\author{
J. Ježek ${ }^{1}$, K. Schulmann ${ }^{1}$, and A. B. Thompson ${ }^{2}$ \\ ${ }^{1}$ Faculty of Science, Praha, Czech Republic \\ ${ }^{2}$ ETH Zuerich, Switzerland
}

Received: 3 January 2001 - Revised: 23 July 2001 - Accepted: 13 August 2001

\begin{abstract}
A model is presented that combines the traditional approach to transpression with the thin viscous sheet approximation of the lithosphere, to study deformation in front of an obliquely colliding indenter. The model produces a zone dominated by lateral simple shear (orogen parallel transcurrent movements) close to the indenter and a zone of dominant pure shear (orogen perpendicular pure shear/thrusting regions) further away from the indenting boundary. The relative width of simple shear dominated zone with respect to the more distant pure shear domain in terms of the angle of convergence is discussed. The possibility of development of transcurrent faults in front of an indenting plate is enhanced by the reactivation of fabric anisotropy created progressively during indentation.
\end{abstract}

\section{Introduction}

The indentation process can be modelled as a collision between stiff (indenting) and weak lithosphere resulting in a deformation of the latter. Two main contrasting types of models exist based on different mechanical behaviour of the lithosphere during continental collision. The first group of models suggests that the lithosphere is composed of undeformable rigid blocks and that shortening is accomodated by localised zones of shearing and lithospheric faults. The second group of models suggests that the weak continental lithosphere behaves mainly as a viscous medium in which the shortening is accomplished by diffuse deformation.

The first group of models are essentially analog-type and try to understand 2D plane geometry of a network of largescale transcurrent faults developed in a layer of viscous material (similar to plasticine) due to indentation of a rigid block (Tapponier and Molnar, 1976; Tapponier et al., 1982; Peltzer and Tapponier, 1988). The main goal of such analog models was to show the geometry of fault patterns in Asia resulting from indentation of the rigid Indian continental indenter. The

Correspondence to: J. Ježek (jezek@natur.cuni.cz) asymmetry of Asiatic fault system was explained by lateral extrusion of mass associated with eastern Pacific subduction. In corresponding analog models, the eastward lateral extrusion associated with northward indentation is modelled using a free mechanical boundary at the eastern side of the experiment and the amount of lateral extrusion is estimated to correspond to $40-60 \%$ of the total deformation (Tapponier et al., 1986; Peltzer and Tapponier, 1988). Davy and Cobbold (1988) produced analog models of indentation using several layers simulating combined brittle and viscous behaviour of deforming lithosphere. These models show that the collisional thickened zone experienced high stress and shortening was associated with extrusion along one major fault, which is a surface expression of viscous deformation at depth. Their model was a compromise between entirely heterogeneous and entirely homogeneous modes of deformation.

The second group of models of lithosphere deformation is mostly numerical. These models are often based on the assumption that the lithosphere deforms on a large cale as a homogeneous and continuous medium with defined integrated viscosity. Some of these models assume that the geometrical boundary conditions, as well as external and internal body forces, are known allowing applications of numerical methods of calculations (England and McKenzie, 1982; Vilotte et al., 1982). There is also a different group of models involving the presence of rheological heterogeneities (Chery et al., 1991; Vauchez et al., 1994; Tomassi and Vauchez, 1997) and showing complex mechanical behaviour of viscous plate during convergence. Most of numerical models of viscous lithosphere deformation don't simulate geological evolution of particular areas but merely testify physical process of indentation. In addition, the numerical models of Houseman and England (1986) suggest that the convergence is mainly absorbed by thickening and that the lateral extrusion does not exceed $30 \%$ of the total shortening (being interpreted as a secondary effect). Broad criticism of numerical models is based on their generally difficult applicability to results of field geology and geophysics (e.g. Witlinger et al., 1998), and on the underestimation of the role of large-scale transcurrent 

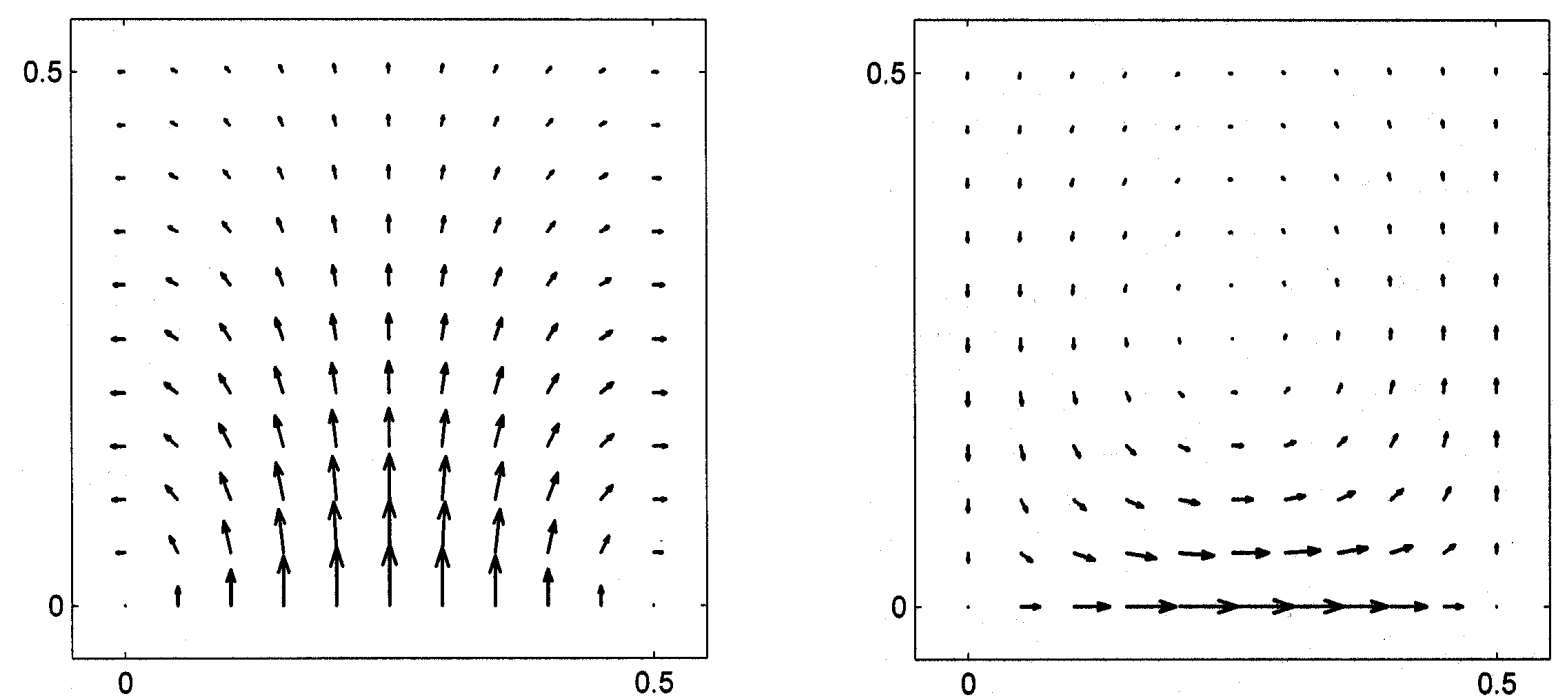

Fig. 1. The velocity field in front of a frontally (a) and tangentially (b) moving indenter. The indenter is approximated by a sinusoidal boundary condition on the lower side of the rectangle with maximum velocity at the middle of the lower side. Computed for the case of Newtonian fluid and Argand number Ar $=0$ from Eq. (20) of England et al. (1985). The wavelength of the sine function applied at the boundary $\lambda=1$ and the arrows showing velocity were scaled so that the maximum length equals the distance between grid points.

faults for lateral extrusion in front of an indenter. Such numerical models of continental collision have been developed in terms of deformation of a thin viscous layer (England and McKenzie, 1982; England et al., 1985). It has been shown that the key role in the indentation process is played by the across strike width of the indenter, whether the type of collision is predominantly of frontal- or lateral-type, together with the viscosity of the foreland. The main result of the work of England et al. (1985) for the model described in the following paragraphs is that deformation in front of the indenter decays nearly exponentially and that the characteristic length-scale for the frontal collision is four times greater than that for a transcurrent regime. This concept was used (England and Houseman, 1986, 1988; Houseman and England, 1986) to explain the mechanics of the Tibetan plateau and to calculate continental deformation of the India-Asia collision zone.

An interesting problem is what kinds of distribution and partitioning of strain would develop for the case of oblique convergence. Structural patterns developed at obliquely convergent plate boundaries are called zones of transpression (Harland, 1971). The effects of oblique convergence are often examined and numerically modeled in a framework of vertical tabular zones confined by steep parallel walls (Sanderson and Marchini, 1984) which resemble weak zones confined by rigid cratons rather than reflecting oblique indentation. In transpressive zones, defined in such a way, the concept of strain partitioning into zones of simple shear deformation and domains of pure shear has become particularly popular (Tikoff and Teyssier, 1994). From observation, we know quite well the geometry of partitioned deformation and consequences for bulk strain in such zones of transpression but the mechanical reasons for strain partitioning are not yet fully understood. In addition, the position of zones of discrete partitioning within transpressional systems are arbitrarily selected (e.g. Tikoff and Green, 1997).

The deformation in front of an obliquely indenting rigid lithosphere can be also described in term of transpression. We combine the traditional transpression model with some equations of thin-sheet deformation from England et al. (1985) to model deformation of zones of oblique indentation. This approach helps us to define zones dominated by lateral simple shear (orogen parallel transcurrent movements) close to the indenter and a zone of dominant pure shear (orogen perpendicular pure shear/thrusting regions) further away from the indenting boundary. This problem was addressed by Vauchez et al. (1994) who discussed the distribution of strain at the termination of a cratonic domain. These authors used finite element modeling to demonstrate the shift of orogen-transverse to orogen-normal tectonic movements around a stiff inclusion which favors strain localisation. In this paper, we discuss the relative width of the simple shear dominated zone with respect to the more distant pure shear domain in terms of angle of convergence assuming no rheological heterogeneity in front of indenter. We also suggest that the strain partitioning, as well as the position of simple shear zone close to indenter margin is an inherent process related to oblique indentation. In addition, the partitioning of deformation due to divergence (non-parallelism) of instantaneous and finite strain in front of an indenter may contribute greatly to the development of transcurrent faults. This concept was used by Tomassi and Vauchez (2001) who applied a model of lattice preferred orientation-induced mechanical anisotropy which may result in directional softening, lead- 
ing to heterogeneous deformation during a subsequent tectonic event. They suggested that during continental rifting, this mechanical anisotropy may induce strain localisation in domains where extensional stress is oblique $\left(30-55^{\circ}\right)$ to the preexisting mantle fabric. The possibility of development of such directional softening in front of an indenting plate is related to reactivation of some fabric anisotropy created progressively during indentation. The faulting originates in areas where strong mechanical anisotropy (finite strain fabric and possibly also frozen lattice preferred orientation of deformation controlling minerals) is at a high angle to the infinitesimal strain axes. In these areas, high shear-strain may develop enabling easy activation of strike-slip movements.

\section{Thin viscous sheet approximation}

The deformation field in front of the indenter was modelled by England et al. (1985); Houseman and England (1986). They used an approximation where the lithosphere was considered as a thin viscous sheet with Newtonian, or nonNewtonian rheology, and developed equations and numerical solutions corresponding to the cases of frontal collision and transcurrent motion of an indenter. The shape of the indenter was represented by a velocity profile applied as the boundary condition. Fig. 1 demonstrates typical features of the velocity field for the case of frontal (Fig. 1a) and transcurrent (Fig. 1b) motion of the indenter showing that the transcurrent velocity decreases with the distance from the indenter much faster than the frontal one. This fact was expressed by England et al. (1985) by approximate formulas for horizontal components of velocity in the central part in front of the indenter. Velocity perpendicular to the boundary (convergent velocity) decreases as

$v(y)=v_{0} \exp \left(-\frac{\pi \sqrt{n}}{2 L} y\right)$

while the velocity parallel to the boundary (transcurrent velocity) also decreases as

$u(y)=u_{0} \exp \left(-4 \frac{\pi \sqrt{n}}{2 L} y\right)$

where $y$ is the distance from the indenter, $v_{0}$ and $u_{0}$ are velocities applied at the boundary of the sheet, $n$ is the power law exponent, and $L$ is the width of the indenter. Therefore the velocities parallel to the boundary decay away more strongly with the distance from the indenter due to the factor 4 in the exponential (Fig. 2). The Eqs. (1) and (2) were developed by England et al. (1985) under the assumption of zero Argand number $(\mathrm{Ar}=0)$, i.e. that the forces arising from variations of crustal thickness are negligible with respect to the forces caused by the colliding indenter, and for a sinusoidal boundary condition. The equations were later verified by Houseman and England (1986) for geologically more reasonable boundary conditions that could be characterized as a rectangle with rounded corners.

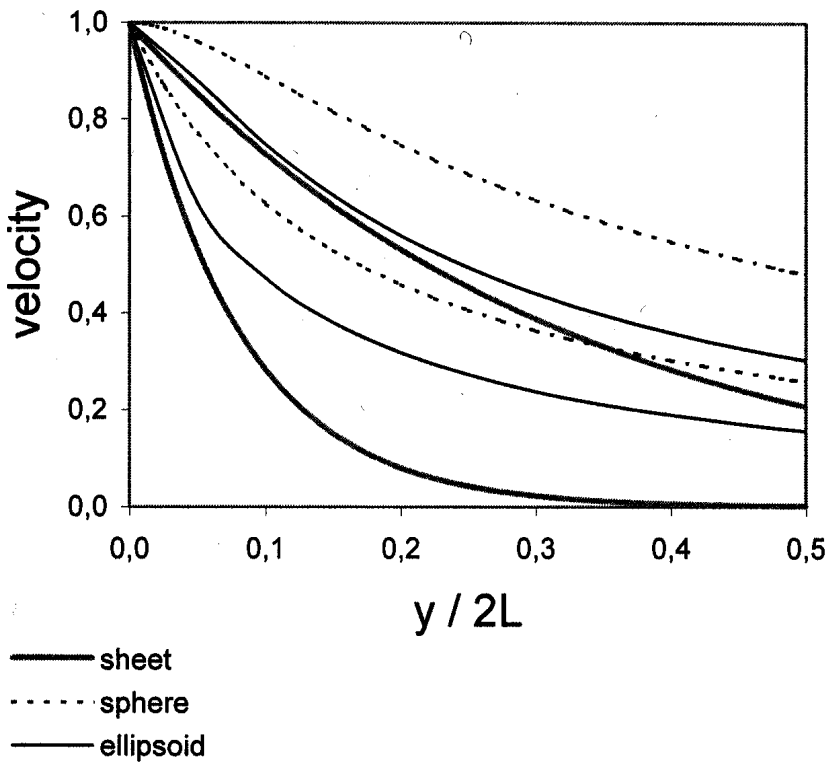

Fig. 2. Comparison of the decrease of convergent and transcurrent velocity across the deformed zone for the thin sheet approximation (thick line), flow around an ellipsoid (thin line) and a flow around sphere (dotted line). The convergent component decreases more slowly with distance from the indenter in all cases, i.e. it is the upper curve for each pair. The distance from the indenter $y$ is normalised to the width of indenter $L$.

\section{Comparison with the flow in front of an ellipsoidal in- denter}

The idea of different attenuation of convergent and transcurrent velocity components is basic to our study on the influence of the obliquity on deformation partitioning. Besides the viscous sheet model, we shall briefly investigate this effect on another - comparative - model and consider viscous flow in front of a rigid ellipsoidal indenter. The governing equations of the problem were found by Oberbeck (1876). From 3D velocity field around an ellipsoid we use only a 2D section. In a $x y$-symmetry plane of an ellipsoid, the components of velocity around the ellipsoid moving along its $x$-axis can be written as

$$
\begin{aligned}
& u(x, y, 0)=V_{x}+\frac{V_{x}}{q_{0}+\alpha_{0} a^{2}}\left\{x \frac{\partial q}{\partial x}-q-\frac{a^{2}}{2} \frac{\partial^{2} \Omega}{\partial x^{2}}\right\} \\
& v(x, y, 0)=\frac{V_{x}}{q_{0}+\alpha_{0} a^{2}}\left\{x \frac{\partial q}{\partial y}-\frac{a^{2}}{2} \frac{\partial^{2} \Omega}{\partial x \partial y}\right\}
\end{aligned}
$$

where $V_{x}$ is the velocity of ellipsoid, $a$ its semiaxis (in the $x$ direction), and all remaining parameters are functions that can be expressed by means of elliptic integrals (see Appendix). Numerical solution of Eqs. (3) and (4) shows that the side velocity field decreases faster than the frontal one, i.e. similarly to the case of a thin sheet approximation, the transcurrent velocity decreases faster that the convergent one with smaller distance from the indenter.

In Fig. 2 we compare transcurrent and convergent components of velocity of the thin sheet approximation to those 


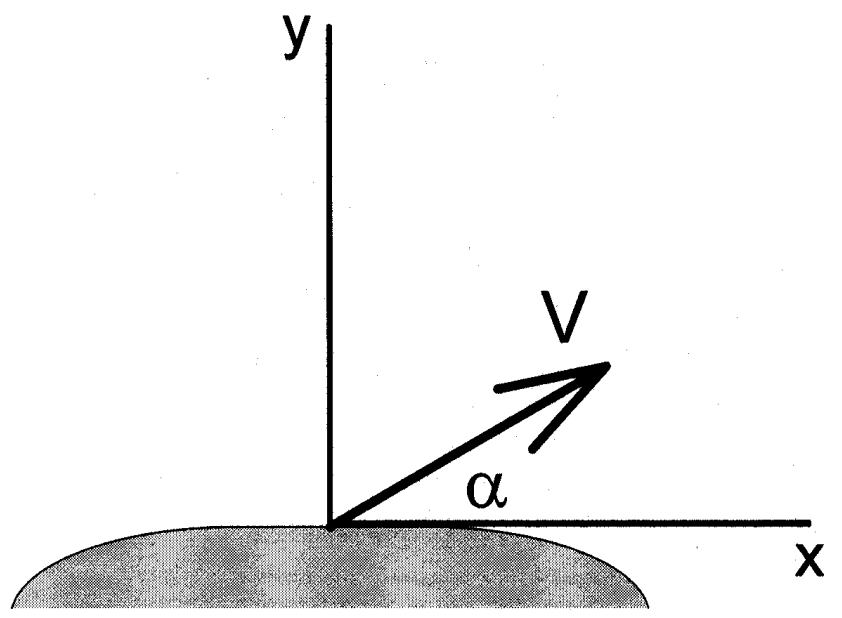

Fig. 3. Coordinate system in front of the indenter: $\alpha$ is the angle of convergence, with $\alpha=90^{\circ}$ for the frontal indentation.

produced by an ellipsoidal indenter. The ellipsoid axial ratios $\mathrm{X}: \mathrm{Y}: \mathrm{Z}$ are 500:100:100 $\mathrm{km}$ for which the velocity field in the $x y$-plane could be comparable to a "viscous sheet-type" indenter of $1000 \mathrm{~km}$ width moving at $1 \mathrm{~cm} / \mathrm{y}$ with parameter $\mathrm{n}=1$, i.e. Newtonian viscosity. In both cases, the velocities exhibit similar decrease near the indenter. This supports the idea that such features should be characteristic for any indentation process. For further comparison we also considered a solution for the flow around a sphere that is known from classical continuum mechanics, e.g. Turcotte and Schubert (1982, p. 263).

\section{The model of strain partitioning based on the thin sheet approximation}

On the basis of different decreases of frontal and transcurrent velocities, we shall now develop a model of partitioning of deformation as a function of obliquity of collision. We shall use the thin visous sheet approximation for the velocities, Eqs. (1) and (2), which have the advantage of a simple form and have built-in the parameter $n$ for the type of rheology. Consider a coordinate system with the $x$-axis horizontal and parallel to the boundary of an indenter colliding obliquely with a weak ductile domain and the $y$-axis horizontal and perpendicular to the boundary (Fig. 3). We shall deal with the velocity field in front of the central part of an indenter where the boundary is assumed to be straight. The indenter moves obliquely with a velocity $V$ and angle of convergence $\alpha$. Similarly as in Thompson et al. (1997), we formally split the velocity $V$ into two components

$v_{0}=V \sin (\alpha)$

$u_{0}=V \cos (\alpha)$

that are perpendicular and parallel to the boundary of the indenter. We assume that these components are boundary values in velocity distribution, and that this distribution has a character of Eqs. (1) for convergent and (2) for transcurrent component, respectively. This means that the decrease of the perpendicular and parallel components of velocity are independent of each other. We therefore have

$v(y)=V \sin (\alpha) \exp \left(-\pi \sqrt{n} \frac{y}{2 L}\right)$
$u(y)=V \cos (\alpha) \exp \left(-4 \pi \sqrt{n} \frac{y}{2 L}\right)$

From these equations we derive the corresponding (nonhomogeneous) distribution of strain rates of contractional strain (pure shear rate) and of tangential shear (simple shear rate) in front of the indenter as

$\dot{\varepsilon}(y)=\frac{\pi \sqrt{n}}{2} \frac{V}{L} \sin (\alpha) \exp \left(-\pi \sqrt{n} \frac{y}{2 L}\right)$

$\dot{\gamma}(y)=2 \pi \sqrt{n} \frac{V}{L} \cos (\alpha) \exp \left(-4 \pi \sqrt{n} \frac{y}{2 L}\right)$

where we omit the minus sign. From Eqs. (9) and (10) it follows that indenters having the same velocity to width ratio, $v / L$, have the same magnitude of strain rates, $\dot{\varepsilon}(0)$ and $\dot{\gamma}(0)$. Any indenter can be, therefore, characterized by the ratio $v / L$ and the angle of convergence $\alpha$. In the course of time, all indenters having the same ratio $v / L$ will produce the same finite deformation at the normalised distance $y / 2 L$ from the indenter.

To describe the deformation history ahead of the indenter we consider a rock sample in the deformed zone moving with the velocity $(u, v)$. As the velocity component $v$ decreases with the $y$-coordinate (it is always less than the velocity of the indenter), the distance of the sample to the indenter will be progressively shortened during time (the sample approaches the indenter boundary). During its motion the sample is subjected to deformation corresponding to the strain rates (9) and (10). If we consider that locally (at any place in front of the indenter) the horizontal shortening is compensated by vertical extrusion without lateral elongation or lateral escape of the material, as, e.g. in the Sanderson and Marchini (1984) model of transpression, we describe instantaneous deformation at the point by the velocity gradient tensor of the form

$\mathbf{L}=\left(\begin{array}{ccc}0 & \dot{\gamma} & 0 \\ 0 & -\dot{\varepsilon} & 0 \\ 0 & 0 & \dot{\varepsilon}\end{array}\right)$

For the case of lateral horizontal escape of the ductile material around the indenter we can use alternatively the velocity gradient tensor of the form

$\mathbf{L}=\left(\begin{array}{ccc}q \dot{\varepsilon} & \dot{\gamma} & 0 \\ 0 & -\dot{\varepsilon} & 0 \\ 0 & 0 & (1-q) \dot{\varepsilon}\end{array}\right)$ 

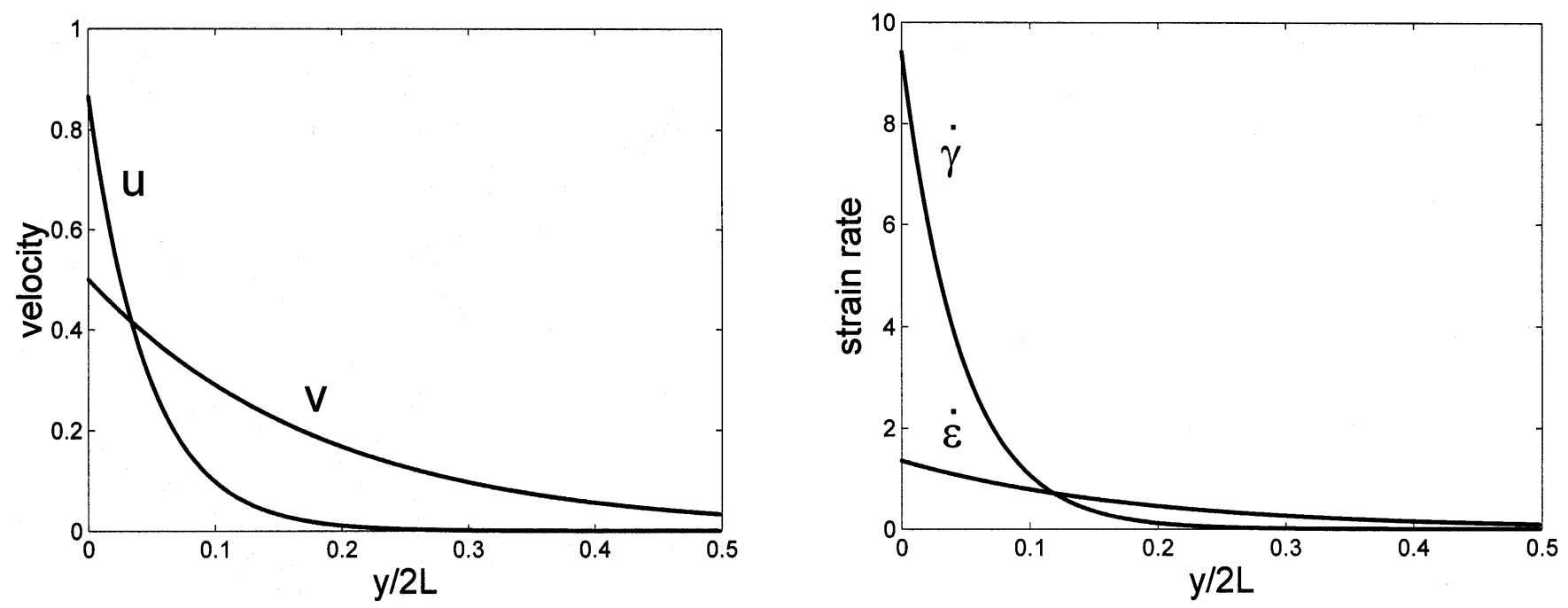

Fig. 4. Oblique indentation with $\alpha=30^{\circ}$, (a) - convergent $(v)$ and transcurrent $(u)$ velocity across the deformed zone, (b) - convergent $(\dot{\varepsilon})$ and transcurrent $(\dot{\gamma})$ strain rates. In the graphs, the velocities (the values on vertical axis) are normalised to the boundary value of velocity $V$ and strain rates to the ratio $V / L$, where $L$ is the width of indenter.

where $q<1$. The instantaneous stretching axes $\dot{s}_{1}>\dot{s}_{2}>\dot{s}_{3}$ can be computed from the stretching tensor (the symmetric part of the velocity gradient tensor).

By integrating instantaneous deformation we compute the accumulation of finite strain in the observed rock sample during time. We characterize the finite strain by conventional strain parameters, namely strain intensity $D$

$D=\sqrt{\ln (X / Y)^{2}+\ln (Y / Z)^{2}}$

and strain symmetry

$K=\frac{\ln (X / Y)}{\ln (Y / Z)}$

where $X, Y, Z$ are semiaxes of finite strain ellipsoid, and further by the orientation of lineation $\left(\mathrm{s}_{1}\right)$ and foliation $\left(\mathrm{s}_{1}-\mathrm{s}_{2}\right)$.

\section{Characteristic features of the model and results of modelling}

The model produces partitioning of ductile deformation that is dependent on the distance from the indenter. In fact we combine two kinds of partitioning. The first one can be seen as partitioning of the intensity of ductile deformation and is expressed by the exponential decay of velocities and strain rates across the deformed zone. The second one is partitioning by the type of deformation (contractional or shear). It is expressed by different attenuation factors in the exponentials and, due to it, the model is also sensitive to the obliquity of collision $(\alpha)$. As an example, we show in Fig. 4 the decrease of perpendicular and parallel components of velocity and corresponding strain rates across the zone ahead of the indenter for an angle of convergence $\alpha=30^{\circ}$. We observe

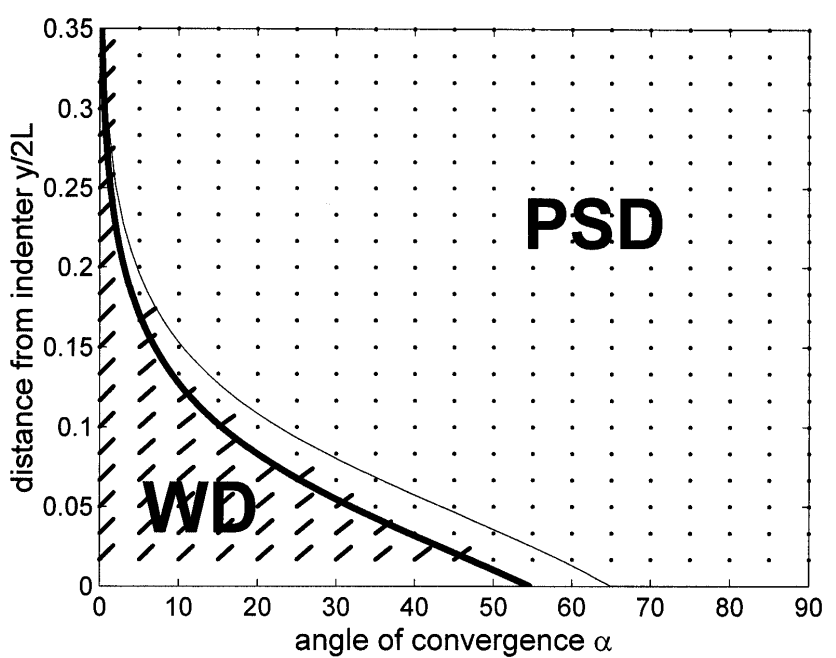

Fig. 5. Transition between WD (wrench dominated) and PSD (pure shear dominated) domains (thick line) as a function of the angle of collision for the system with no side flow (velocity gradient tensor of Eq. (11)). Below the transition-curve, in the WD domain the orientation of first instantaneous strain axis (stretching axis) is horizontal, at $45^{\circ}$ to the indenter boundary (marked by strokes) and above in PSD domain it is vertical (points). The thin line indicates a shift of the transition-curve when side flow is allowed (velocity gradient tensor Eq. (12), parameter $q=0.25$ ).

that in a relatively narrow zone near the indenter, the transcurrent velocity is greater than the frontal one. This pattern also applies to the strain rates.

Analyzing the eigenvectors and eigenvalues associated with the instantaneous deformation tensor we find the instantaneous stretching axes $\dot{s}_{1}>\dot{s}_{2}>\dot{s}_{3}$. We shall use the term "instantaneous lineation" for the direction defined by the first instantaneous stretching axis $\left(\dot{s}_{1}\right)$ and the term "in- 

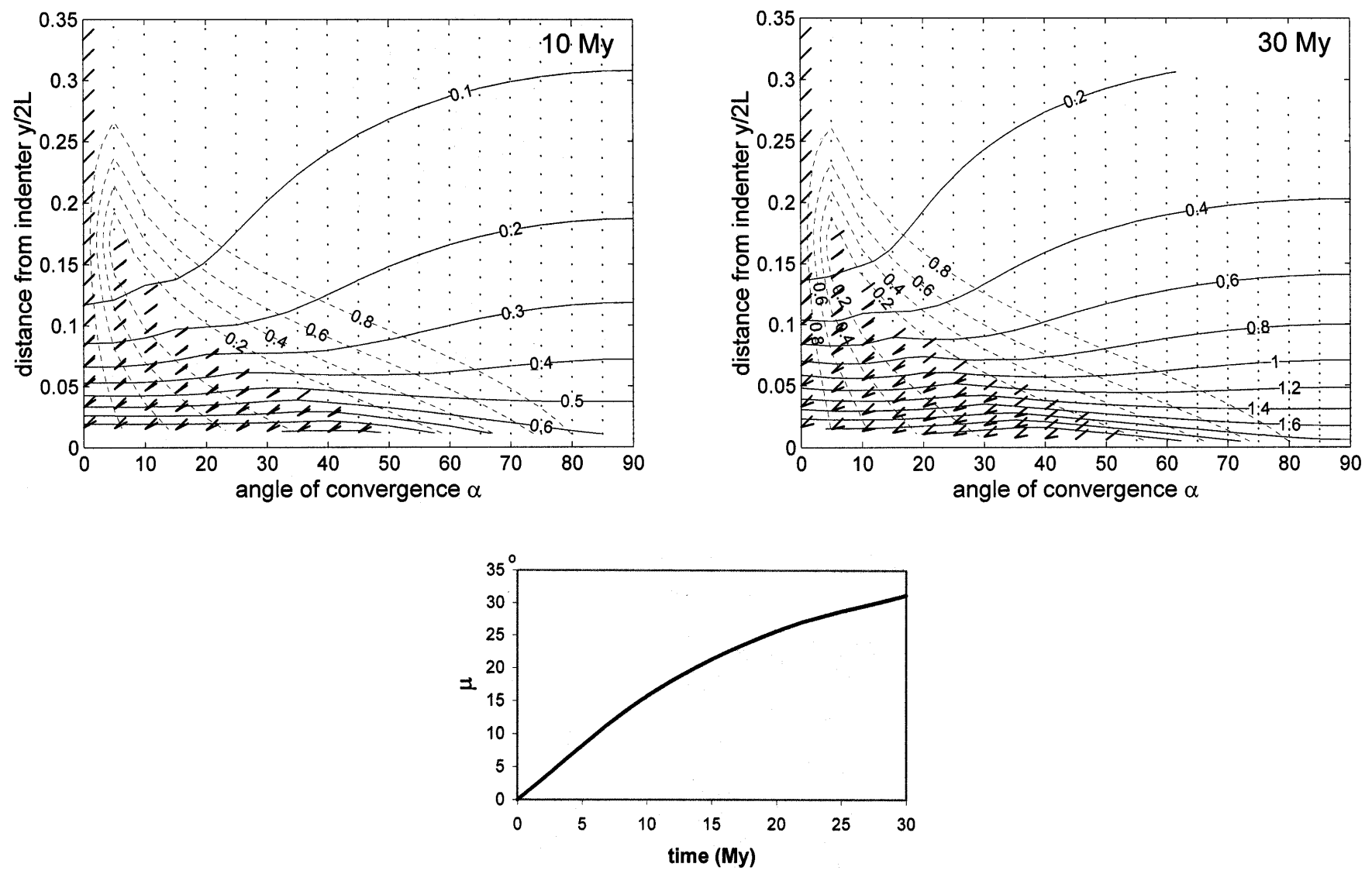

Fig. 6. Temporal development of finite strain parameters along a $y$-profile in front of the indenter as a function of the angle of collision: (a) after $10 \mathrm{My}$, (b) - after $30 \mathrm{My}$ of indentation. The intensity of finite strain $D$ decreases progressively with the distance from indenter (solid lines), the symmetry parameter $K$ creates a pattern almost constant in time (dashed lines). Initially equi-distant spaced points on $y$-profiles approach the indenter during indentation. In the PSD zone (see Fig. 5), the developed finite lineation is vertical (dots at observed points). In the WD domain (see Fig. 5), the developed finite horizontal lineation (lower stroke at each point) is almost parallel to the first instantaneous strain axis (upper stroke at each point) for $10 \mathrm{My}$, and diverts from it progressively, as seen for $30 \mathrm{My}$. The strokes of horizontal lineation indicate also a projection of foliation on the horizontal $x y$-plane. (c) - the development of the degree of misorientation between instantaneous and finite strain through time, for a sample located near the indenter boundary. The angle of convergence $\alpha=20^{\circ}$. The sample was initially at $100 \mathrm{~km}$ distance $(y / 2 L=0.017)$ and after $30 \mathrm{My}$ at $66 \mathrm{~km}(y / 2 L=0.011)$ from the indenter.

stantaneous foliation" for the plane defined by the first and second ones $\left(\dot{s}_{1}-\dot{s}_{2}\right)$. For our model it is typical to expect a near-indenter zone with horizontal instantaneous lineation (wrench-dominated, WD) and a distant zone with vertical instantaneous lineation (pure-shear-dominated, PSD). In case of no lateral escape of the material, the transition between the zones appears approximately at the distance

$y_{T}=\frac{2 L \ln [1.41 \cot (\alpha)]}{2 \pi \sqrt{n}}$

from the indenter. Note that this distance is somewhat less than the distance at which the contractional and shear strain rates equal each other. In Fig. 5 we show the transition between WD and PSD domains for all possible angles (thick line), with the assumption of no lateral escape of the material and for non-Newtonian rheology with the power-law exponent $n=3$. The larger the angle of convergence $(\alpha)$ the shorter is the WD domain. Note that theoretically narrow
WD domains are possible for angles to about $50^{\circ}$. Below the transition-curve (Fig. 5), in the WD domain the orientation of the instantaneous lineation is horizontal (marked by strokes on Fig. 5) and above in the PSD domain it is vertical (marked by points). This characterisitic of the instantaneous deformation indicates that in a consequent development, at lesser distances from the indenter, shearing parallel to the indenter boundary prevails over contraction, and a horizontal finite lineation (i.e. the lineation defined by the first axis of the finite strain ellipsoid) can develop and would be maintained during at least a part of the deformation process. The model would typically produce narrow domains near the indenting boundary where shear deformation is accumulated, and wider zones far away from the indenter dominated by the contractional deformation.

In Fig. 6 we compute the temporal development of finite strain parameters along a $y$-profile across the domain ahead of an indenter of $3000 \mathrm{~km}$ width moving with a velocity of 

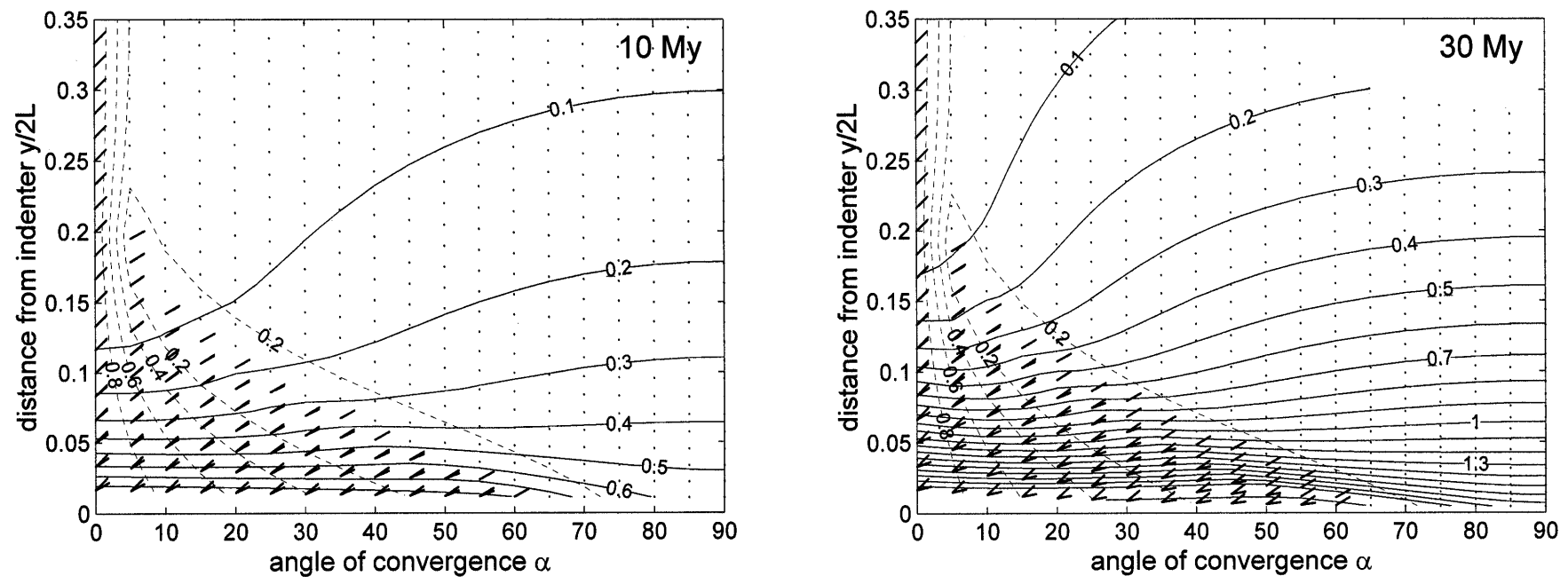

Fig. 7. Temporal development of finite strain parameters in front of the indenter for the case when a part of the convergent component is compensated by a horizontal side flow along the indenter: (a) - after $10 \mathrm{My}$, (b) - after $30 \mathrm{My}$ of indentation. Velocity gradient tensor of Eq. (12) with $q=0.25$ was used. Compare with Fig. 6.

$5 \mathrm{~cm} /$ year (velocity-width ratio $v / L=5 / 300 \mathrm{My}^{-1}$ ) for different angles of convergence $(\alpha)$. In Fig. 6a we observe the situation after $10 \mathrm{My}$ of indentation. For angles of convergence $(\alpha)$ higher than about $50^{\circ}$, the deformation has a pure shear character, the intensity of finite strain $D$ decreases progressively with the distance from indenter, and lineation (the finite strain ellipsoid maximum axis) is vertical. For low angles of convergence, we observe in the WD domain a development of finite horizontal lineation that is almost parallel to the instantaneous lineation. This is valid also for the developed foliation. At the distance corresponding to the transition between WD and PSD domains (see Fig. 5, the thick curve) the finite strain ellipsoids are of the flattening-type (strain parameter $K$ is near zero values). Furthermore, the finite lineation switches to vertical which becomes practically observable (higher values of $K$ ) at larger distances where pure shear predominates. Fig. $6 \mathrm{~b}$ shows the continuation of the history after $30 \mathrm{My}$. The WD and PSD domains kept their character and the only difference is that the horizontal finite lineation and foliation in the WD domain becomes subparallel to the indenter boundary so that the angle to the instantaneous lineation increases. Fig. 6 shows that the wrench dominated region may show observable horizontal finite lineations $(K>0.2)$ at a distance of $300 \mathrm{~km}(Y / 2 L=0.05)$ and $200 \mathrm{~km}$ $(Y / 2 L=0.03)$ from the indenter margin with angles of convergence of $20^{\circ}$ and $30^{\circ}$, respectively. The shape of the finite strain ellipsoid is not strongly dependent on the duration of indentation as shown by contours of the $K$ parameter in strain maps, as in Figs. 6a, b. However, the strain intensity parameter, $D$, is strongly dependent on time and also on the angle of convergence $(\alpha$, for distances from the indenting plate greater than $400 \mathrm{~km}(Y / 2 L>0.07)$. We note that the strain intensities after $10 \mathrm{Ma}$ of indentation are very low at any distance from the indenting plate, but after $30 \mathrm{Ma}$ become important in a region $400 \mathrm{~km}$ wide adjacent to the indenter boundary. Here, the finite strain intensities became similar to those recorded in continental crust elsewhere (Pfiffner and Ramsay, 1982). In addition, the contours of $D$ in this region are almost independent of the convergence angle $(\alpha)$. This implies, that the fabric intensity close to the indenter boundary does not discriminate the convergence angle, while the ellipsoid shape and orientation of stretching lineation near the indenter are more characteristic. Fig. 6 shows also the relationship of mutual orientation between finite and instantaneous foliations (we denote this angle by $\mu$ ) in the WD region. As noted by Tikoff and Teyssier (1994) the finite and instantaneous strain ellipsoids are substantially non-parallel during transpressional deformation. Fig. 6a demonstrates that after $10 \mathrm{Ma}$ of indentation the misorientation between finite strain field and instantaneous strain is still negligible. However, after $30 \mathrm{Ma}$ of indentation the misorientation angle $(\mu)$ becomes important, reaching angles higher than $30^{\circ}$. As an example, we show in Fig. 6c, the degree of misorientation between instantaneous and finite strain through time, for a sample located near to the indenter boundary. The degree of misorientation is strongly dependent on convergence angle $(\alpha)$ so that the domain where the misorientation angle $\mu \sim 30^{\circ}$ rapidly decreases with angle of convergence $(\alpha)$ approaching $40^{\circ}$ in Fig. 6 . We note also that $\mu$ is developed in areas with a dominant plain-strain type of ellipsoid, or flattening, but always with horizontal lineation.

If we allow a part of the contractional component to be compensated by a horizontal side flow (lateral escape) along the indenter, the situation does not change substantially. The WD zone becomes wider as it is marked in Fig. 5 (thin line) where we considered the velocity gradient tensor of the form of Eq. (12) with $q=0.25$ (lateral escape of $25 \%$ of material). In Fig. 7 we show the corresponding time development 

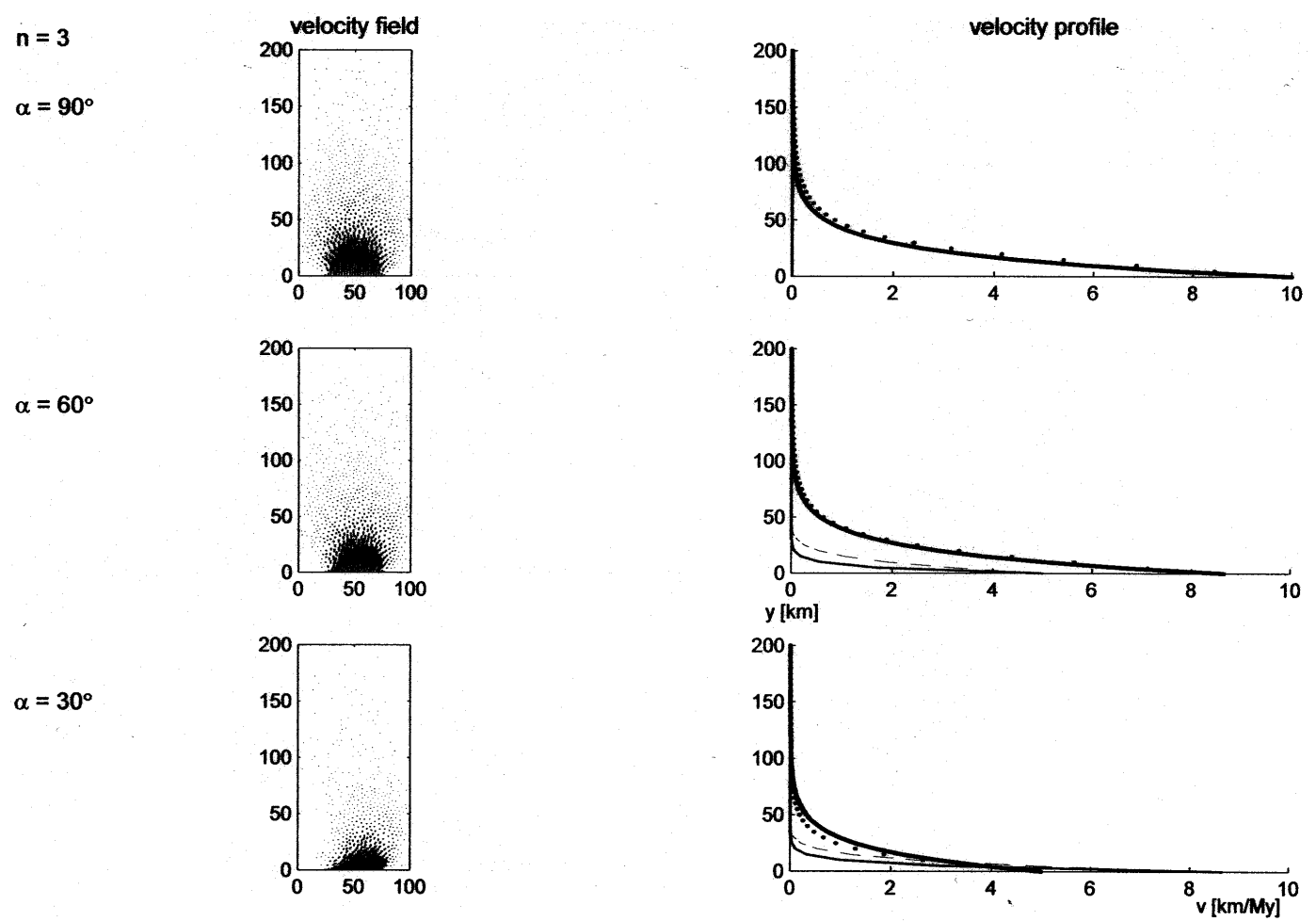

Fig. 8. Comparison of the velocity profiles computed by the finite-element method and their approximations. Left column - velocity fields computed for the case of non-Newtonian fluid, $n=3$, and Argand number Ar $=0$ from Eq. (20) of England et al. (1985). The indenter is represented by the boundary condition on the lower boundary of the domain, the angles of convergence $\alpha=90^{\circ}$, $60^{\circ}$, and $30^{\circ}$. Right column - corresponding convergent (dotted line) and transcurrent (dashed line) velocity profiles in front of the indenter compared to the approximations of Eq. (7) (thick solid line) and Eq. (8) (thin solid line).

after 10 and $30 \mathrm{My}$. The only difference with respect to the previous case without lateral escape (Fig. 6) is that the WD zone exists even for angle of convergence, $\alpha=65^{\circ}$. The degree of misorientation of $\mu$ is apparent at distance from the indenting plate with $Y / 2 L=0.07$ and in a narrow zone close to indenter boundary for angle of convergence of $50^{\circ}$.

\section{Discussion}

\subsection{Model assumptions}

The model is built as a linear combination of the expressions for the decrease of the velocity components. For the case of a Newtonian viscous sheet with zero Argand number, it is possible to show that a sum of the solutions for frontal and transcurrent components (e.g. Eqs. (23) and (24) in England et al., 1985) fulfill the equation of motion (England et al., 1985, Eq. (20)). Similarly, the flow around an ellipsoidal indenter, our Eqs. (3) and (4), is a solution of a linearized NavierStokes equation for a Newtonian viscous fluid (Stokes flow) and, thus, any combination of velocity fields fulfilling these equations and boundary conditions is also a solution. Therefore, in the case of a Newtonian viscous fluid and $\mathrm{Ar}=0$, it is justified to split the velocity of the indenter into two components (Eqs. (5) and (6)) to obtain frontal and transcurrent velocity profiles.
A possible source of inaccuracy is the use of Eqs. (1) and (2) for approximation of the frontal and transcurrent velocity profiles in the central part in front of the indenter. Some measure of the errors introduced by this approximation was evaluated by England et al. (1985) (see Eqs. (33) and (34), and relevant discussion in their paragraph 4) showing that the Eqs. (1) and (2) can be considered as a first order approximation of velocity in front of the indenter.

Another problem is that, in case of non-Newtonian viscous fluid $(n>1)$, the solutions are no longer additive (equations of motion are not linear) and we have to deal with another error produced by neglecting this fact. We have investigated these errors by finite-element modeling, based on a general equation for horizontal components of velocity (England et al., 1985, Eq. (18)), and we found that they do not exceed errors arising from the approximate equations (1) and (2). As an example we show in Fig. 8 the results of finite-element modeling demonstrating that, also in the nonNewtonian case, our approximations (Eq. (7) and (8)) are in agreement with the finite-element solution.

In sum we can conclude that a partitioning which is dependent on the type (contractional, wrenching) of deformation should play a determinant role in the development of the deformation field in front of the indenter and the use of our approach is justified as a first order approximation. 


\subsection{Tectonic implications}

In this work we have modeled partitioning of deformation developed in a homogeneous viscous zone in front of an obliquely moving rigid indenter. Our model shows that strain partitioning, i.e. the existence of a zone of lateral shearing displacing material in front of an obliquely indenting plate is an inherent mechanism of any collisional process. The zone of wrench dominated and intense ductile deformation is located close to the indenter, and is dominated by simple shear. In contrast, a zone of weak pure shear dominated deformation develops further from the indentation front. The width of such zones of dominant simple shear is dependent on the angle of convergence $(\alpha)$ and can exist even for a convergence angle close to $50^{\circ}$. If lateral escape is added, as a process controlled by a free lateral boundary, then WD zones exist for even larger values of the angle of convergence $(\alpha)$.

The geometrical relationships between finite and instantaneous strain have important consequences for the possible development of strike slip partitioning in front of an obliquely indenting plate. In the course of time, the degree of finite anistropy in deforming rocks close to the indenter, becomes rather high and the planar fabric becomes parallel to the orientation of the indenter boundary. However, the instantaneous stretching axis $\dot{s}_{1}$ is oriented at a high angle to the rigid plate boundary and forms an angle $\mu$ close to or higher than $30^{\circ}$ with a finite strain fabric. The instantaneous stretching tensor has similar significance as does a stress tensor for development of faulting and reactivation of preexisting anisotropies. Therefore, a sufficiently high angle between finite strain anisotropy and instantaneous $\dot{s}_{1}$ axis may generate high resolved shear stress on pre-existing planes and may be responsible for activation of strike-slip faults. Thus, the reactivation of previously developed anisotropy by orogen parallel transcurrent faulting is very likely in areas adjacent to indenting plates. This directional softening (Vauchez and Tommasi, 2001) associated with previously developed fabrics may control the propagation of the initial instability and development of large scale transcurrent faults parallel to the indenter boundary.

The effects of strain discrete displacement partitioning in transpressive zones, where part or all of the lateral displacements are accommodated by discrete zone-boundary-parallel faults have been considered by some authors (Jones and Tanner, 1995; Teyssier et al., 1995; Tikoff and Teyssier, 1994). However, the location of simple shear zones or strike slip faults within the transpressional zone was chosen rather arbitrarily, often in the centre of a zone (Jones and Tanner, 1995; Dewey et al., 1998) or, more rarely, at the margins of transpressional zones (Tikoff and Teyssier, 1994). It is quite evident from the above cited works, that the problem of locations, periodicity and spatial distribution of heterogeneous simple shear dominated zones within the global transpressional system are not yet sufficiently solved. Our work proposes an explanation for the position of simple shear dominated zones at the margin of an indenter which is in agreement with natural observations in active transpressional systems. In addition, the width of pure shear and simple shear dominated zones and the amount of partitioning are controlled by the angle of convergence and the creep exponent. In contrast, the amount of strain partitioning in previous models was arbitrarily selected to demonstrate the role of orogen parallel slip on the bulk strain (see, e.g. Dewey et al., 1998).

The applicability of our model to natural examples is complex. It is not our intention to specifically model, for example, the indentation of India into Asia in this paper, but rather to show the potential of models of oblique indentation which may be tested on both lithospheric and smaller crustal scales. On the lithospheric scale, we suggest the possibility of development close to the indenter front of wrench dominated high strain zones, the width of which is controlled by across-strike length of the indenting plate and the angle of convergence. Similar results were obtained by Vauchez and Tommasi (1994); Tommasi and Vauchez (1997) for rheologically heterogeneous lithosphere including a craton within a weak continent which favors strain localization, initiation of continental-scale shear zones, and differential vertical deformations. Our model assumes that the lithosphere in front of the indenting plate is rheologically and thermally homogeneous allowing undisturbed distribution of deformation in the far field. On this scale we can define the ratio between width of a remote zone of pure shear dominated strain and the wrench dominated zone, which may help to define the convergence angle in cases of fossil oblique indentation.

On a smaller scale, the oblique indentation of rigid crustal blocks within weak sediments may also be appropriate to explain superposed cleavage fronts (Lexa and Schulmann, 1999). The validation of a numerical model with respect to kinematic constraints of basement blocks and cleavage-fold relationships is based on regional mapping of cleavage patterns and finite strain patterns in weak metasedimentary formations. There is a range of natural examples in which the basement (composed of gneisses and granites) behaves as a rigid promontory of irregular shape with respect to weakly metamorphosed mechanically weak slates (Sintubin, 1999; Lexa and Schulmann, 1999). In agreement with Woodcock et al. (1988) and Mansy et al. (1999) the cleavage-fold patterns in such deformable weak rocks reflects the geometry and direction of movement of rigid blocks. For instance, the example of Caledonian regional shortening in Britain associated with northward movement of the Midlands Promontory shows decrease of deformation intensity from the margin of the indenting block (Woodcock et al., 1988), which is similar to that produced in the model of thin viscous sheet deformation. In addition, the wrench dominated shear zones are well developed at obliquely oriented boundaries of the Midland Promontory (e.g. Sintubin, 1999, Fig. 10). Similar pattern is developed along the Cantabrian promontory in France as described from a range of field studies (e.g. Brun and Burg, 1982; Burg et al., 1987). Also, we suggest that rotated strain trajectories developed close to the Main Central Thrust of the Himalaya (Brun et al., 1985) may not result from superposed simple shear components, but may be better approximated 
using our approach. However, the field studies describing quantitatively the development of natural strain gradients in relation to promontory shape and direction of movement are rather scarce and limited to the boundary regions only (e.g. Dias and Ribeiro, 1994).

We are aware that rigorous quantitative geometrical analysis of deformation within weaker zones including fault kinematics and displacement rates on various scales is needed to make our models directly applicable. On such small scales, the vertical boundary condition for a rigid indenter has to be replaced by an inclined (thrust) surface (Ellis et al., 1995) which may generate triclinic symmetry of deformation and result in partitioning of strain on thrust faults.

\section{Conclusions}

We are aware that our model does not describe completely complex geological situations in collisional zones and the values of computed strain parameters can only be taken as a first order approximation. Nevertheless, it shows some important aspects of oblique collision.

1) For angles of convergence from 0 to about $50^{\circ}$, the model produces a relatively narrow belt near the indenting boundary with prevailing wrench deformation while the contractional deformation diffuses to larger distances from the indenter. This fact is in good agreement with natural observations of narrow shear zones bordering collisional domains.

2) A comparison with a homogeneous model of transpression is very interesting. Tikoff and Teyssier (1994) show that in the Sanderson and Marchini (1984) model of transpression, a WD deformation is possible for an angle of convergence $\alpha<20^{\circ}$. In the course of deformation, due to the more efficient accumulation of the contractional component of strain, the horizontal lineation progressively shortens and finally switches to a vertical lineation. Note that classical transpression is homogeneous and therefore the lineation is similar throughout the whole domain. In contrast to this, our model exhibits an across-domain partitioning of vorticity which corresponds to a stepwise transition from horizontal to vertical lineation and can produce horizontal lineations also for angles $\alpha>20^{\circ}$.

3) The character of the instantaneous deformation field in front of the indenter changes only little through time. The width of WD zone remains nearly constant through time if the angle of convergence $(\alpha)$ does not change.

4) Through time, a foliation develops near to the indenter boundary that forms an initial angle $45^{\circ}$ and then becomes progressively subparallel to the indenter boundary. This asymmetry of fabric can interact with instantaneous stretching and be a source of possible directional softening and strike-slip faulting in the course of the indentation process. In the framework of our models, such effects are possible for angles of convergence from 0 to about $50^{\circ}$.

Acknowledgement. The research was supported financially by the Grant Agency of Charles University (210/2000/B) and by the Grant Agency of Czech Republic (205/98/004). We are grateful to two anonymous reviewers for very helpful reviews of the manuscript.

We thank Basil Tikoff for reminding us about the standard usage of terminology, namely:

\begin{tabular}{lllll} 
Stress & & Strain & & Movement \\
\cline { 1 - 1 } \cline { 5 - 6 } Compressional & & Contractional & & Convergent \\
Tensional & & Extensional & & Divergent \\
Tangential & & Shear & & Transcurrent
\end{tabular}

\section{Appendix}

Velocity field around an ellipsoidal indenter

Governing equations of the flow around an ellipsoid were found by Oberbeck (1876). Here, we present them in somewhat different notation. Consider a point in the fixed coordinate system outside the moving ellipsoid and denote by $L$ a positive root of the equation

$\frac{x^{2}}{a^{2}+\lambda}+\frac{y^{2}}{b^{2}+\lambda}+\frac{z^{2}}{c^{2}+\lambda}=1$.

Further, let us put

$$
\Delta(\lambda)=\sqrt{\left(a^{2}+\lambda\right)\left(b^{2}+\lambda\right)\left(c^{2}+\lambda\right)}
$$

and

$\Omega=\int_{L}^{\infty}\left(\frac{x^{2}}{a^{2}+\lambda}+\frac{y^{2}}{b^{2}+\lambda}+\frac{z^{2}}{c^{2}+\lambda}-1\right) \frac{d \lambda}{\Delta(\lambda)}$,

$\alpha=\int_{L}^{\infty} \frac{d \lambda}{\left(a^{2}+\lambda\right) \Delta(\lambda)}$,

$q=\int_{L}^{\infty} \frac{d \lambda}{\Delta(\lambda)}$.

If $L=0$, the quantities $\alpha, q$ are denoted by $\alpha_{0}, q_{0}$. The components of the velocity field around an ellipsoid moving along the $x$-axis (Oberbeck 1876, Eq. (43)) are then expressed in our notation as

$$
\begin{aligned}
& u(x, y, z)=V_{x}+\frac{V_{x}}{q_{0}+\alpha_{0} a^{2}}\left\{x \frac{\partial q}{\partial x}-q-\frac{a^{2}}{2} \frac{\partial^{2} \Omega}{\partial x^{2}}\right\}, \\
& v(x, y, z)=\frac{V_{x}}{q_{0}+\alpha_{0} a^{2}}\left\{x \frac{\partial q}{\partial y}-\frac{a^{2}}{2} \frac{\partial^{2} \Omega}{\partial x \partial y}\right\},
\end{aligned}
$$


$w(x, y, z)=\frac{V_{x}}{q_{0}+\alpha_{0} a^{2}}\left\{x \frac{\partial q}{\partial z}-\frac{a^{2}}{2} \frac{\partial^{2} \Omega}{\partial x \partial z}\right\}$,

where $V_{x}$ is the velocity of ellipsoid. From Eq. (A1) we find

$\frac{\partial \lambda}{\partial x}=\frac{2 x P^{2}}{a^{2}+\lambda}$

where

$\frac{1}{P^{2}}=\frac{x^{2}}{\left(a^{2}+\lambda\right)^{2}}+\frac{y^{2}}{\left(b^{2}+\lambda\right)^{2}}+\frac{z^{2}}{\left(c^{2}+\lambda\right)^{2}}$.

Using differentation of the integral (A5) with respect to a parameter we obtain

$$
\begin{aligned}
\frac{\partial q}{\partial x} & =\frac{\partial}{\partial x} \int_{L}^{\infty} \frac{d \lambda}{\Delta(\lambda)}= \\
& \int_{L}^{\infty} \frac{\partial}{\partial x} \frac{d \lambda}{\Delta(\lambda)}-\frac{1}{\Delta(L)} \frac{\partial L}{\partial x}=-\frac{2 x P^{2}}{\left(a^{2}+L\right) \Delta(L)}
\end{aligned}
$$

and similarly

$$
\begin{aligned}
& \frac{\partial q}{\partial y}=-\frac{2 y P^{2}}{\left(b^{2}+L\right) \Delta(L)}, \\
& \frac{\partial q}{\partial z}=-\frac{2 z P^{2}}{\left(c^{2}+L\right) \Delta(L)} .
\end{aligned}
$$

Further, the derivatives of $\Omega$ may be expressed as

$$
\begin{aligned}
& \frac{\partial^{2} \Omega}{\partial x^{2}}=2 a-\frac{4 x^{2} P^{2}}{\left(a^{2}+\lambda\right)^{2} \Delta(\lambda)}, \\
& \frac{\partial^{2} \Omega}{\partial x \partial y}=-\frac{4 x y P^{2}}{\left(a^{2}+\lambda\right)\left(b^{2}+\lambda\right) \Delta(\lambda)}, \\
& \frac{\partial^{2} \Omega}{\partial x \partial z}=-\frac{4 x z P^{2}}{\left(a^{2}+\lambda\right)\left(c^{2}+\lambda\right) \Delta(\lambda)} .
\end{aligned}
$$

Therefore, only elliptic integrals $\alpha$ and $q_{0}$ (Eqs. (A4) and (A5)) are needed to evaluate the velocity field, Eqs. (A6) to (A8). A procedure for the integral (A4) was described in Ježek et al. (1999). The procedure for integral (A5) can be found in a similar way.

\section{References}

Brun, J. P. and Burg, J. P.: Combined thrusting and wrenching in the Ibero-Armorican arc - a corner effect during continental collision, Earth and Planetary Science Letters, 61, 319-332, 1982.

Brun, J. P., Burg, J. P., and Ming, C. G.: Strain trajectories above the main central thrust (Himalaya) in southern Tibet, Nature, 313, 388-390, 1985.

Burg, J. P., Bale, P., Brun, J. P., and Girardeau, J.: Stretching lineation and transport direction in the Ibero-Armorican arc during the Siluro-Devonian collision, Geodinamica Acta, 1, 71-87, 1987.
Chery, J., Vilotte, J. P., and Daignieres, M.: Thermomechanical evolution of a thinned continental lithosphere under compression implications for the Pyrenees, J. Geophys. Res., 96, 4385-4412, 1991.

Davy, P. and Cobbold, P.: Indentation tectonics in nature and experiment, 1. experiments scaled by gravity, Bull. Inst. Uppsala, 14, 143-162, 1988.

Dewey, J. F., Holdsworth, R. E., and Strachan, R. A.: Transpression and transtension zones, in: Holdsworth, R. E., Strachan, R. A., and Dewey, J. F. (Eds.): Continetal transpression and transtensional tectonics, Geological Society, London, Special publications, 135, 1-14, 1998.

Dias, R. and Ribeiro, A.: Constriction in a transpressive regime an example in the Iberian branch of the Ibero-Armorican arc, J. Struct. Geol., 16, 1543-1554, 1994.

Ellis, S.: Forces driving continental collision: reconciling indentation and mantle subduction tectonics, Geology, 24, 699-702, 1996.

England, P. and McKenzie, D.: A thin viscous sheet model for continental deformation, J. R. Astr. Soc., 70, 295-321, 1982.

England, P. and Houseman, G.: Finite strain calculations of continental deformation, 2. comparison with the India-Asia collision zone, J. Geophys. Res., 91, 3664-3676, 1986.

England, P., Houseman, G., and Sonder, L.: Length scales for continental deformation in convergent, divergent, and strike-slip environments: Analytical and approximate solutions for a thin viscous sheet model, J. Geophys. Res., 90, 3551-3557, 1985.

Harland, W. B.: Tectonic transpression in Caledonian Spitsbergen, Geological Magazine, 108, 27-42, 1971.

Houseman, G. and England, P.: Finite strain calculations of continental deformation: 1 . Method and general results for convergent zones, J. Geophys. Res., 91, 3651-3663, 1986.

Ježek, J., Saic., S., Segeth, K., and Schulmann, K.: Threedimensional hydrodynamical modelling of viscous flow around a rotating ellipsoidal inclusion, Computers and Geosciences, 25, 547-558, 1999.

Jones, R. R. and Tanner, P. W. G.: Strain partitioning in transpression zones, J. Struct. Geol., 17, 793-802, 1995.

Lexa, O. and Schulmann, K.: Creatceous evolution of the Variscan basement of the SE Western Carpathians: Combination of continental underthrusting and indentation tectonics, Geologica Carpathica, 50, 119-120, 1999.

Mansy, J. L., Everaerts, M., and De Vos, W.: Structural analysis of the adjacent Acadian and Variscan fold belts in Belgium and northern France from geophysical and geological evidence, Tectonophysics, 309, 99-116, 1999.

Oberbeck, A.: Über stationäre Flüssigkeitsbewegungen mit Berücksichtigung der inneren Reibung, Crelle, 81, 62-80, 1876.

Peltzer, G., and Tapponnier, P.: Formation and evolution of strike slip faults, rifts, and basins during India-Asia collision: an experimental approach, J. Geophys. Res., 93, 15 085-15 117, 1988.

Pfiffner, O. A. and Ramsay, J. G.: Constrains on geological strain rates: arguments from finite strain states of naturally deformed rocks, J. Geophys. Res., 87, B1, 311-321, 1982.

Sanderson, D. J. and Marchini, W. R. D.: Transpression, J. Struct. Geol., 6, 449-548, 1984.

Sintubin, M.: Arcuate fold and cleavage patterns in the southeastern part of the Anglo-Brabant Fold Belt (Belgium): tectonic implications, Tectonophysics, 309, 81-97, 1999.

Tapponier, P. and Molnar, P.: Slip-line field theory and large-scale continental tectonics, Nature, 264, 319, 1976. 
Tapponnier, P., Peltzer, G., Armijo, R., Le Dain, A. Y. , and Cobbold, P.: Propagation extrusion tectonics in Asia: new insight from simple experiments with plasticine, Geology, 10, 611-616, 1982.

Tapponnier, P., Peltzer, G., and Armijo, R.: On the mechanics of the collision between India and Asia, in: Ramsay, J. G., Coward, M. P., and Ries A. C. (Eds.): Collision Tectonics, Geological Society, London, Special publications, 19, 1986.

Teyssier, Ch., Tikoff, B., and Markley, M.: Oblique plate motion and continental tectonics, Geology, 23, 447-450, 1995.

Tikoff, B. and D. Greene, Stretching lineations in transpressional shear zones: An example from the Sierra Nevada Batholith, California. J. Struct. Geol., 19, 29-39, 1997.

Tikoff, B. and Teyssier, Ch.: Strain modelling of displacementfield partitioning in transpressional orogens, J. Struct. Geol., 16, 1575-1588, 1994.

Thompson, A. B., Schulmann, K., and Ježek, J.: Thermal evolution and exhumation in obliquely convergent (transpressive) orogens, Tectonophysics, 280, 171-184, 1997.

Tommasi, A. and Vauchez, A.: Continental-scale rheological heterogeneities and complex intraplate tectono-metamorphic pat- terns: insights from a case-study and numerical models, Tectonophysics, 279, 327-350, 1997.

Tommasi, A. and Vauchez, A.: Continental rifting parallel to ancient collisional belts: an effect of the mechanical anisotropy of the lithospheric mantle, Earth and Planetary Science Letters, 185, 199-210, 2001.

Turcotte, D. L. and Schubert., G.: Geodynamics, John Willey, New York, 450 pp., 1982.

Vauchez, A., Tommasi, A., and Egydiosilva, M.: Self-indentation of a heterogeneous continental lithosphere. Geology 22, 967-970, 1994.

Vilotte, J. P., Daignieres, M., and Madariaga, R.: Numerical modelling of intraplate deformation: simple mechanical models of continental collision, J. Geophys. Res., 87, 10, 709-728, 1982.

Witlinger, G. P., Tapponier, G., Poupinet, J., Mei, J., Danian, S., Herquel G., and Masson, F.: Tomographic evidence for localized shear along the Altyn Tagh fault, Science, 282, 74-76, 1998.

Woodcock, N. H., Awan, M. A., Johnson, T. E., Mackie, A. H., and Smith, R. D. A.: Acadian tectonics of Wales during Avalonia Laurentia convergence, Tectonics, 7, 483-495, 1988. 